\title{
A Theoretical Framework to Formalize AGI-Hard Problems
}

\section{Pedro Demasi*}

\author{
Jayme L Szwarcfiter $^{* \dagger}$
}

\author{
Adriano J O Cruz ${ }^{\dagger}$
}

\begin{abstract}
The main goal of the Artificial General Intelligence field (AGI) to create "human level intelligence" is known as a very ambitious one (Hut04). On the way to the field development there are many difficult problems to solve, like natural language translation, for example, which seem to share some "hardness" properties. The terms "AI-Complete" and "AIHard", by analogy with the terms "NP-Complete" and "NPHard" from computational complexity theory (CLRS01), have been informally used to classify them although there are also works that propose some kind of formal definition (SA07), (vABHL03). This work proposes a theoretical framework with formal definitions to distinguish these problems and discuss its use in practical applications and how their properties can be used in order to achieve improvements in the AGI field.
\end{abstract}

\section{Introduction}

In order to achieve a human level intelligence, it is clear that many "human" problems must be solved on the way to it. Some tasks, like natural language understanding, are easy to be solved by humans. Even other tasks which may require some kind of prior training, like text translation between two natural languages, are also routinely solved by humans.

The lack of formal definition for such problems is still a huge barrier for computationally solving them. This work proposes a formal framework to classify which problems can be considered as human-level intelligence bounded and which can not.

We will thus distinguish the problems in these different classes:

- Non AGI-Bound: problems that are not of AGI interest. Although they may be "hard" in computational sense, they are not in the scope of AGI study.

- AGI-Bound: problems that require some kind of humanlevel intelligence to be properly solved.

\footnotetext{
* Universidade Federal do Rio de Janeiro, Cidade Universitaria, Programa de Engenharia de Sistemas e Computacao, Bloco H, Rio de Janeiro/RJ, Brazil, 21941-972

${ }^{\dagger}$ Universidade Federal do Rio de Janeiro, Cidade Universitaria, Nucleo de Computacao Eletronica, Rio de Janeiro/RJ, Brazil, 20001-970
}

- AGI-Hard: problems that are at least as hard as any AGIBound problem.

\section{Human Solvers and Human Oracles}

The boolean set $\mathbb{B}$ will be used as $\mathbb{B}=\{0,1\}$ and $\mathbb{B}=$ $\{$ no, yes $\}$ interchangeably without any loss of generality.

Definition 1. A solver is a black box which can solve any problem a Turing Machine (TM) or a human can solve, using constant time. Formally, a solver will be a function $f_{s}: \mathbb{N} \rightarrow \mathbb{N}$ such that given an input $x \in \mathbb{N}$ it will give an output $y \in \mathbb{N}$ as the answer. The output $y$ given by the solver is called an acceptable answer to the input $x$.

Definition 2. We say that $y \in \mathbb{N}$ is an acceptable output for input $x \in \mathbb{N}$ if, and only if, $\exists f_{s}$ such that $f_{s}(x)=y$.

Definition 3. We say that $y \in \mathbb{N}$ is an incorrect output for input $x \in \mathbb{N}$ if, and only if, $\nexists f_{s}$ such that $f_{s}(x)=y$.

Definition 4. An oracle to a problem $\mathcal{P}$ is a function $f$ : $\mathbb{N} \times \mathbb{N} \rightarrow \mathbb{B}$.

Definition 5. A valid oracle for a problem $\mathcal{P}$ will always answer yes to an input pair $(x, y)$ if $y$ is a correct output for $x$. It will always answer no to an input pair $(x, y)$ if $y$ is an incorrect output for $x$. For every pair $(x, y)$ such that $y$ is an acceptable output for $x$, but not correct, the oracle may answer either yes or no.

Definition 6. The set $\Omega^{\mathcal{P}}$ is the set of all valid oracles for the problem $\mathcal{P}$.

Definition 7. We say that $x \in \mathbb{N}$ is a valid input for oracle $f: \mathbb{N} \times \mathbb{N} \rightarrow \mathbb{B}$ if, and only if, $\exists y \in \mathbb{N}, f(x, y)=1$.

Definition 8. For a given problem $\mathcal{P}, y \in \mathbb{N}$ is a correct output for input $x \in \mathbb{N}$ if, and only if, $\forall f \in \Omega^{\mathcal{P}}, f(x, y)=$ 1. This definition implies that every correct output is also an acceptable one.

\section{Properties and Operation of Valid Oracles and their Sets}

Definition 9. Two oracles are equal, $f_{a}=f_{b}$ if, and only if, $\forall x, y \in \mathbb{N}, f_{a}(x, y)=f_{b}(x, y)$

Definition 10. The intersection of two oracles $f_{a}$ and $f_{b}$, represented as $f_{c}=f_{a} \cap f_{b}$, is $f_{c}=f_{a}(x, y) \wedge$ $f_{b}(x, y) \forall x, y \in \mathbb{N}$ 
Definition 11. The union of two oracles $f_{a}$ and $f_{b}$, represented as $f_{c}=f_{a} \cup f_{b}$, is $f_{c}=f_{a}(x, y) \vee f_{b}(x, y) \forall x, y \in \mathbb{N}$

Property 1. The set $\Omega^{\mathcal{P}}$ of valid oracles over problem $\mathcal{P}$ is closed under intersections and unions.

Definition 12. A master oracle $f_{\Omega}$ for a problem $\mathcal{P}$ is the union of all elements of $\Omega^{\mathcal{P}}$. Thus $f_{\Omega}=\bigcup_{f \in \Omega^{\mathcal{P}}} f$.

Definition 13. A trivial oracle $f_{\emptyset}$ for a problem $\mathcal{P}$ is the intersection of all elements of $\Omega^{\mathcal{P}}$. Thus $f_{\emptyset}=\bigcap_{f \in \Omega^{\mathcal{P}}} f$.

Definition 14. Let $\overline{f_{a}}$ be the complement of $f_{a}$, it is such that $f_{a} \cup \overline{f_{a}}=f_{\Omega}$ and $f_{a} \cap \overline{f_{a}}=f_{\emptyset}$.

Property 2. The set $\Omega^{\mathcal{P}}$ of valid oracles over problem $\mathcal{P}$ is closed under complement.

Definition 15. We say that $f_{a} \geq f_{b}$ (meaning that $f_{a}$ dominates $f_{b}$ ) if, and only if, for every valid input $x \in \mathbb{N}$ of $f_{b}$, $\forall y \in \mathbb{N}, f_{a}(x, y)=f_{b}(x, y)$

Property 3. Mutual dominance between oracles is such that $f_{a} \geq f_{b}, f_{b} \geq f_{a} \leftrightarrow f_{a}=f_{b}$

\section{AGI-Boundness and AGI-Hardness}

Definition 16. The cardinality of $\Omega^{\mathcal{P}}$ is such that $\left|\Omega^{\mathcal{P}}\right|=$ $\prod_{x=1}^{|\mathbb{N}|} 2^{\left|\mathcal{A}_{x}^{\mathcal{P}}\right|}=2^{\sum_{x=1}^{|\mathbb{N}|}\left|\mathcal{A}_{x}^{\mathcal{P}}\right|}$

Definition 17. A problem $\mathcal{P}$ is Non AGI-Bound if, and only if, $\left|\Omega^{\mathcal{P}}\right|=1$

Definition 18. Let the set $\mathcal{A}_{x}^{\mathcal{P}}$ be such that $\forall y \in \mathcal{A}_{x}^{\mathcal{P}}$, $f_{\Omega}(x, y) \otimes f_{\emptyset}(x, y)=1$ ( $\otimes$ means logical exclusive-or).

Definition 19. A problem $\mathcal{P}$ is AGI-Bound if, and only if, $\left|\Omega^{\mathcal{P}}\right|>1$

Theorem 1. If the summation of cardinalities of $\mathcal{A}^{\mathcal{P}}$ is equal to the cardinality of $\mathbb{N}$, then the cardinality of $\Omega^{\mathcal{P}}$ is equal to $\mathbb{R}$, that is $\sum_{x=1}^{|\mathbb{N}|}\left|\mathcal{A}_{x}^{\mathcal{P}}\right|=|\mathbb{N}| \rightarrow\left|\Omega^{\mathcal{P}}\right|=|\mathbb{R}|$

Corolary 1. If the there is at least one $x \in \mathbb{N}$ such that the cardinality of $\mathcal{A}_{\mathcal{P}}^{\mathcal{P}}$ is equal to the cardinality of $\mathbb{N}$, then the cardinality of $\Omega^{\mathcal{P}}$ is equal to $\mathbb{R}$, that is $\exists x \in \mathbb{N},\left|\mathcal{A}_{x}^{\mathcal{P}}\right|=$ $|\mathbb{N}| \rightarrow\left|\Omega^{\mathcal{P}}\right|=|\mathbb{R}|$

Corolary 2. Let the set $\mathcal{B}=\left\{x,\left|\mathcal{A}_{x}^{\mathcal{P}}\right|>0\right\},|\mathcal{B}|=|\mathbb{N}| \rightarrow$ $\left|\Omega^{\mathcal{P}}\right|=|\mathbb{R}|$

Using Definition 16, we have by Theorem 1 that $\left|\Omega^{\mathcal{P}}\right|=$ $2^{|\mathbb{N}|}=|\mathbb{R}|$. A simple way to see that $2^{|\mathbb{N}|}=|\mathbb{R}|$ is to realize that this is equivalent to have a countable infinite number of binary digits. Thus we can write any number of $\mathbb{R}$ in binary base, and therefore we have an one-to-one correspondence.

Definition 20. A problem $\mathcal{P}$ is AGI-Hard if, and only if, $\left|\Omega^{\mathcal{P}}\right|=|\mathbb{R}|$

Definition 21. Given an oracle $f: \mathbb{N} \times \mathbb{N} \rightarrow \mathbb{B}$ and a problem $\mathcal{P}$, return yes if $f \in \Omega^{\mathcal{P}}$ and no otherwise.

Definition 22. Given problems $\mathcal{P}$ and $\mathcal{Q}$ we define as weak dominance $\mathcal{P} \succeq \mathcal{Q} \leftrightarrow \exists f \in \mathcal{P}, \forall f^{\prime} \in \mathcal{Q}, f \geq f^{\prime}$

Definition 23. Given problems $\mathcal{P}$ and $\mathcal{Q}$ we define as strong dominance $\mathcal{P} \geq \mathcal{Q} \leftrightarrow \forall f \in \mathcal{P}, \forall f^{\prime} \in \mathcal{Q}, f \geq f^{\prime}$

Theorem 2. A condition for a problem that is not dominated $\exists x \in \mathbb{N},\left|\mathcal{A}_{x}^{\mathcal{P}}\right|>1 \rightarrow \nexists \mathcal{Q}, \mathcal{Q} \geq \mathcal{P}$
Theorem 2 says that if a problem $\mathcal{P}$ has at least one input with more than one strictly acceptable output then there is no problem $\mathcal{Q}$ such that $\mathcal{Q}$ strongly dominates $\mathcal{P}$.

When we are dealing with traditional computational complexity we are usually concerned with the amount of basic steps our algorithm will perform in function of the size of the input. For AGI-Bound, however, the complexity can be measured in terms of the problem ambiguity, which is the size of the valid oracle set, $|\Omega|$.

Of course even if we can deal with ambiguity reduction there will be still the need of some time and space eficiently. It would be worthless if we are able to solve an AGI-Hard problem but not in an acceptable time frame or if we have no storage capacity for it. These concerns become more important when implementing any methods of AGI-Hard problems and there is the need for further development in this area also.

\section{Further Work and Conclusion}

This work presented a new theoretical framework to formally define what AGI problems are, which are "hard" problems and briefly outlined a way of interpreting AGI problems complexity.

Some points that will be adressed in future works will be a definition of "AI" problems on the set of Non AGIBound problems as dominated problems by AGI-Hard ones, the link between those kind of problems and a better definition of their relations.

AGI problems complexity development should be a great help to better solving some AGI-Hard problems, and the results of experimental application will show that.

The use of human computation in light of the theoretical model presented in this paper should also be investigated as it still can lead to very encouraging results.

Is summary, we believe that the theoretical framework introduced in this paper, and to be further and deeper developed in future works, contributes to the AGI field research, helping to improve practical results and stimulating novel theoretical discussions.

\section{References}

[CLRS01] T. Cormen, C. Leiserson, R. Rivest, and C. Stein. Introduction to Algorithms. MIT Press, second edition, 2001.

[Hut04] M. Hutter. Universal Artificial Intelligence: Sequential Decisions based on Algorithmic Probability. Springer, Berlin, 2004.

[SA07] D. Shahaf and E. Amir. Towards a theory of aicompleteness. In 8th International Symposium on Logical Formalizations of Commonsense Reasoning, 2007.

[vABHL03] L. von Ahn, M. Blum, N. Hopper, and J. Langford. Captcha: Using hard ai problems for security. In In Proceedings of Eurocrypt, pages 294-311. SpringerVerlag, 2003. 